\title{
Computation of Shortest Path in a Fuzzy Network: Case Study with Rajasthan Roadways Network
}

\author{
P.K.De \\ Associate Professor \\ Department of Mathematics \\ National Institute of Technology \\ Silchar- 788010 (Assam), India
}

\author{
Amita Bhinchar \\ Assistant Professor \\ Department of Mathematics \\ Rajdhani Institute of Technology and Management \\ Jaipur-303904, India
}

\begin{abstract}
This paper propose a shortest path problem with fuzzy parameters in the domain of Operations Research which is based on Bellman Dynamic Programming algorithm. Attention has been paid to the study of fuzzy network with topological ordering.. Here we discuss the shortest path problem from a specified vertex to all other vertices in a network. For illustration a real life example has been considered from Rajasthan State Roadways Transport Network.
\end{abstract}

General Terms : Shortest Path

Keywords : Shortest path, Weighted graph, Triangular fuzzy number, Bellman dynamic programming..

\section{INTRODUCTION}

The shortest path problem (SPPP) is one of the most fundamental and well-known combinatorial optimization problems that appears in many applications as a sub-problem. The lengths of arcs in the network represents travelling time, cost, distance or other variables. In real life applications, these arc lengths could be uncertain and to determine the exact value of these arc lengths is very difficult or sometimes difficult for decision maker. In such a situation fuzzy shortest path problem (FSPP) seems to be more realistic, where the arc lengths are characterized by fuzzy numbers. While determining a shortest path in such a fuzzy environment we required ranking of fuzzy numbers [1,2,3]. In 1980 Dubois and Prade [4] first introduce fuzzy shortest path problem.. Okada and Soper [5] developed an algorithm based on multiple labeling approach which is useful to generate number of non-dominated paths. Applying fuzzy min concept they have introduced an order relation between fuzzy numbers. Applying extension principle Klein [ 6], has given an algorithm which results dominated path on a acyclic network.

In this paper attention has been paid to the study of determination of shortest path in a Fuzzy network by applying dynamic programming approach. The main objective of this paper is to determine the shortest path from a source to a destination in a network where the edge weights are uncertain. Bellman dynamic programming technique is applied to determine the shortest path and where the edge weights are characterized by triangular fuzzy numbers. For comparison of fuzzy numbers fuzzy ranking technique has been adopted as discussed by Yao and $\mathrm{Wu}$ [1].

\section{2.}

\section{DEFINITIONS}

AND

\section{PRELEMINARIES}

In this section, some definition and preliminaries concerning the fuzzy shortest path problems and ranking method are introduced.

Let $X=\{x\}$ be a universe, i.e. the set of all possible (feasible, relevant) elements to be considered. Then a fuzzy set (or a fuzzy subset) $\mathrm{A}$ in $\mathrm{X}$ is defined as a set of ordered pairs $\mathrm{A}=\left\{\left(\mathrm{x}, \boldsymbol{\mu}_{\mathrm{A}}(x)\right) / \mathrm{x} \in \mathbf{X}\right\}$ and $\mu_{\mathrm{A}}: \mathrm{X} \rightarrow[0,1]$ is the membership function and $\boldsymbol{\mu}_{\mathrm{A}}(x)$ is the membership grade or degree of association of $\mathrm{x}$ in A., where 0 value indicates non belongingness and 1 indicates full belongingness.

Definition 1: The triangular fuzzy number A denoted by $A=(a, b, c)$, is a fuzzy set defined on $R$ with the membership function defined as

$$
\begin{aligned}
\mu_{A}(x) & =0, \quad x<a \\
& =(x-a /(b-a), \quad a \leq x \leq b \\
& =(c-x) /(c-b), \quad b \leq x \leq c \\
& =0, \quad x>c
\end{aligned}
$$




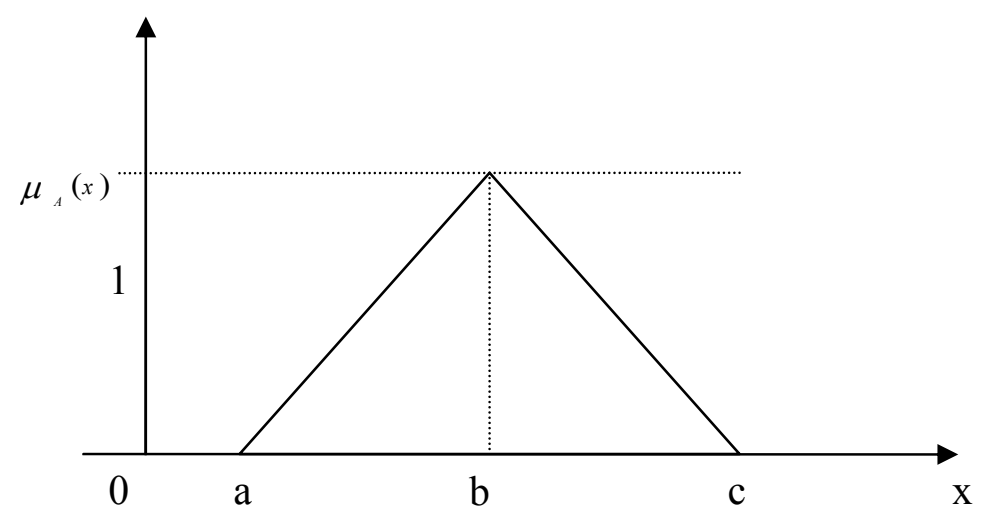

Fig. 1.Traingular fuzzy number

Definition 2: For each $\widetilde{d}=(a, b, c ;) \in F \quad$.the signed distance of $\widetilde{d}$ measured from 0 is defined by $d(\tilde{d}, 0)=\frac{1}{4}(2 b+a+c)$.

Property 1: Let $\quad \widetilde{A}=(a, b, c) \quad$ and $\widetilde{B}=(p, q, r) \in F$.then we obtain the binary operation $d(\widetilde{A} \oplus \widetilde{B}, 0)=d(\widetilde{A}, 0)+d(\widetilde{B}, 0)$.

Definition 3: Let $\quad \widetilde{A}=(a, b, c) \quad$ and $\widetilde{B}=(p, q, r) \in F$. The ranking of fuzzy number $F$ are defined by

$$
\begin{gathered}
\widetilde{A} \prec \widetilde{B} \text { iff } d(\widetilde{A}, 0)<d(\widetilde{B}, 0) \\
\widetilde{A} \approx \widetilde{B} \text { iff } d(\widetilde{A}, 0)=d(\widetilde{B}, 0)
\end{gathered}
$$

\section{Example:1}

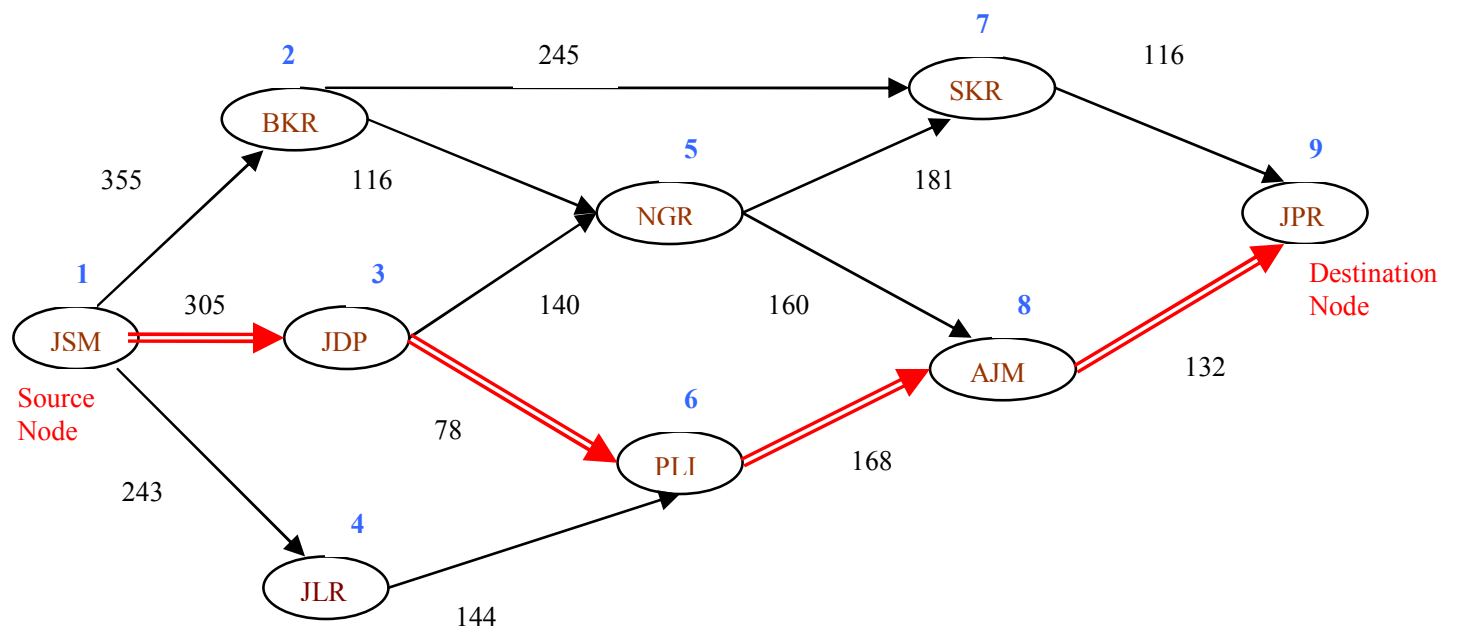

Fig.2. The Distance Network

Name of the cities with city codes which we are going to use in our Network.

\begin{tabular}{|c|c|c|c|c|c|c|}
\hline Node No & Name of City & City Code & Node No & Name of City & City Code \\
\hline 1 & JAISALMER & JSM & 6 & PALI & PLI \\
2 & BIKANER JODHPUR & BKR & 7 & SIKAR & SKR \\
3 & JALORE & JDP & & 8 & AJMER & AJM \\
\hline
\end{tabular}




\begin{tabular}{|l|l|l|l|l|l|l|}
\hline 4 & NAGAUR & JLR & & 9 & JAIPUR & JPR \\
5 & & NGR & & & \\
\hline
\end{tabular}

\section{BELLMAN DYNAMIC PROGRAMMING FORMULATION FOR SHORTEST PATH}

According to Bellman's equation ,a DP formulation for the shortest path problem can be given as follows : Given a network with an acyclic directed graph $G=(V, E)$ with n vertices numbered from 1 to $n$ such that 1 is the source and $n$ is the destination. Then we have

$f(n)=0$

$f(i)=\min _{i<j}\left\{d_{i j}+f(j) \mid\langle i, j\rangle \in E\right\}$

Here $\boldsymbol{d}_{i j}$ is the weight of the directed edge $\langle i, j\rangle$, and $f(i)$ is the length of the shortest path from vertex $i$ to vertex $n$.

From fig2 and equation (1), the solution of DP can be derived as follows:

$f(9)=0, \quad f(8)=$

$\min _{8<j}\left\{d_{8 j}+f(j) \mid<8, \mathrm{j}>\in E\right\}=d_{89}=132$

$f(7)=\min _{7<j}\left\{d_{7 j}+f(j) \mid<7, \quad \mathrm{j}>\in E\right\}$

$=d_{79}=116$,

$f(6)=\min _{6<j}\left\{d_{6 j}+f(j) \mid<6, \quad \mathrm{j}>\in E\right\}$

$=d_{68}+d_{89}=300$, $f(5)=\min \left\{d_{57}+f(7), d_{58}+f(8)\right\}=$ $\min \{181+116,160+132\}=292$

Using the same process we have $f(4)=444$, $f(3)=378, f(2)=361$. finally, $\mathrm{j}=1$; see the row $\mathrm{I}=$ 1.Since there are three entries , $f(1)=\min$ $\left\{d_{12}+f(2), d_{13}+f(3), d_{14}+f(4)\right\}=$ $\min \{355+361,305+378,243+444\}=683$. in summary ,the shortest path obtained from $=d_{13}+f(3)=d_{13}+d_{36}+f(6)=d_{13}+d_{36}+$ $\boldsymbol{d}_{68}+f(8)=\boldsymbol{d}_{13}+\boldsymbol{d}_{36}+\boldsymbol{d}_{68}+\boldsymbol{d}_{89}$ is $1,3,6,8,9$ with length 683 .

\section{COMPUTATION OF SHORTEST- PATH IN FUZZY NETWORK}

In this problem we consider is that the edge weight in the network denoted by $\boldsymbol{d}_{i j}$ and the edge weight should be expressed using fuzzy linguistics, and also this used in triangular fuzzy number.

$$
\widetilde{d}_{i j}=\left(d_{i j}-\delta_{i j 1}, d_{i j}, d_{i j}+\delta_{i j 2}\right),
$$

Where $0<\delta_{i j 1}<d_{i j}, \delta_{i j 2}>0$ Since $\delta_{i j 1}$ and $\delta_{i j 2}$ should be determined by the DM. 


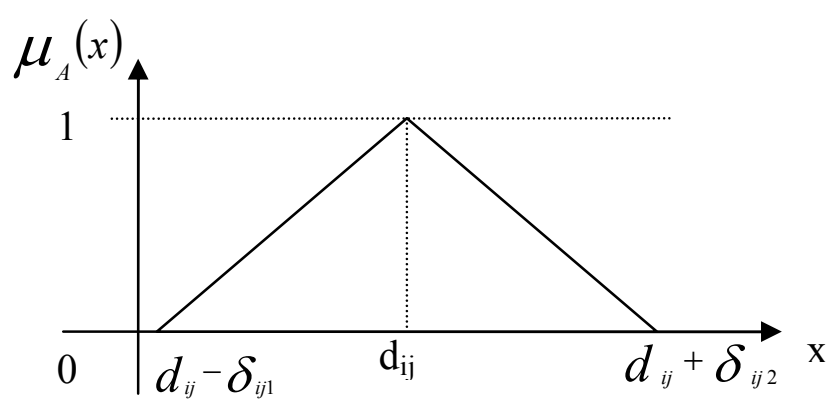

Fig. 3. The fuzzy number

From the above definition of sign distance, we obtain

$$
d\left(\widetilde{d}_{i j}, 0\right)=d_{i j}+\frac{1}{4} \delta_{i j}=d_{i j}^{\circ}
$$

where $\delta_{i j}=\delta_{i j 2}-\delta_{i j 1}$. This is the signed distance of $\widetilde{d}_{i j}$ measured from 0. since

$d_{i j}^{\circ}=d\left(\widetilde{d}_{i j}, 0\right)=\frac{1}{4}\left[4 d_{i j}+\delta_{i j 2}-\delta_{i j 1}\right]=$

$d_{i j}+\frac{1}{4}\left(\delta_{i j 2}-\delta_{i j 1}\right)>0$,we conclude that $d\left(\widetilde{d}_{i j}, 0\right)$ is a positive distance measured from 0 to $\widetilde{d}_{i j}$ and $d_{i j}^{\circ}$ is also a positive number measured from 0. if $\delta_{i j 1}=\delta_{i j 2}$, then we obtain $d_{i j}^{\circ}=d_{i j}$. Thus the fuzzy problem becomes crisp. We call $d_{i j}^{*}=d_{i j}+\frac{1}{4} \delta_{i j}$ an estimate of the edge weight $\langle i, j\rangle$ in the fuzzy sense.

Because there are finite path from node 1 to mode $\mathrm{n}$ in a network, there must exit a path $\mathrm{P}=\left\langle i, \boldsymbol{i}_{1}, \boldsymbol{i}_{2}, \ldots \boldsymbol{i}_{m(i)}, n\right\rangle \quad$ for $f(i)=$ $d_{i_{i_{1}}}{ }^{+} d_{i_{1} i_{2}}{ }^{+\ldots . .+} d_{i_{m(i)}{ }^{n}}$. Note that $f(i)$ is the length of the shortest path from $i$ to vertex $n$.We then derive inequalities from $f(i)$ as

$$
d_{i_{1}}+d_{i_{1} i_{2}}+\ldots d_{i_{m(i)} n} \leq d_{i_{k_{1}}}+d_{k_{1} k_{2}}+\ldots d_{k_{p(k)}{ }^{n}}
$$

Where at least one equal sign holds for all possible paths, $P=\left\langle i, k_{1}, k_{2}, \ldots, k_{p(k)}, n\right\rangle$,from vertex $i$ to vertex $n$.in summary, this is $f(i)=$ $\min \left\{d_{k_{1}}+d_{k_{1} k_{2}}+\ldots d_{k_{p(k)}{ }^{n}} \mid\right.$ forP $\left.=\left\langle i, k_{1}, k_{2}, \ldots k_{p(k)}, n\right\rangle\right\}$

The DM should choose appropriate values for parameters to satisfy

$$
\delta_{i i_{1}}+\delta_{i_{1} i_{2}}+\ldots+\delta_{\left.i_{m(i)}\right)^{n}} \leq \delta_{i k_{1}}+\delta_{k_{1} k_{2}}+\ldots+\delta_{k_{p(k)} n}
$$

Adding one quarter of (3) and (4), we obtain

$$
d\left(\widetilde{d}_{i_{1}} \oplus \widetilde{d}_{i_{1} i_{2}} \oplus \ldots \oplus \widetilde{d}_{i_{m(i)} n}\right) \leq d\left(\widetilde{d}_{i_{1}} \oplus \widetilde{d}_{k_{1} k_{2}} \oplus \ldots \oplus \widetilde{d}_{k_{p(k)} n}\right)
$$

Where at least one equal sign holds. we see that( 4) is equivalent to

$\widetilde{d}_{i_{i_{1}}} \oplus \widetilde{d}_{i_{1} i_{2}} \oplus \ldots \oplus \tilde{d}_{i_{m(i)}{ }^{n}} \leq \widetilde{d}_{i_{k_{1}}} \oplus \widetilde{d}_{k_{1} k_{2}} \oplus \ldots \oplus \widetilde{d}_{k_{p(k)}}$ 
Where at least one $\approx$ holds for all possible paths from vertex $i$ to vertex $n$.Obviously, (5) is obtained from fuzzifying (2) and taking (3) as a fuzzifled condition. Note that

There with the given ranking definition we can write

$$
\begin{aligned}
& d\left(\widetilde{d}_{i_{i_{1}}} \oplus \tilde{d}_{i_{1} i_{2}} \oplus \ldots \oplus \tilde{d}_{i_{m(i)}{ }^{n}}, 0\right)= \\
& d\left(\widetilde{d}_{i_{i_{1}}}, 0\right)+d\left(\widetilde{d}_{i_{1} i_{2}}, 0\right)+\ldots+d\left(\widetilde{d}_{i_{m(i)}{ }^{n}}, 0\right) \\
& =d_{i_{i_{1}}}{ }^{+} d_{i_{1} i_{2}}{ }^{+\ldots}+d_{i_{m(i)}{ }^{n}} .
\end{aligned}
$$

similarly, we obtain

$$
\begin{aligned}
& d\left(\tilde{d}_{i k_{1}} \oplus \tilde{d}_{k_{1} k_{2}} \oplus \ldots \oplus \tilde{d}_{k_{p(k)}{ }^{n}}, 0\right)=d_{i k_{1}}^{\circ} \\
& +d_{k_{1} k_{2}}^{\circ}{ }^{+\ldots+} d_{k_{p(k)}{ }^{n}}^{\circ}
\end{aligned}
$$

Then from(1a ), ( 5) and (6), we derive the following inequalities:

$d_{i i_{1}}^{\circ}+d_{i_{1} i_{2}}^{\circ}+\ldots+d_{i_{m(i)}{ }^{n}}^{\circ}$

$\leq \cdot d_{{ }_{i} k_{1}}^{\circ}+d_{k_{1} k_{2}}^{\circ}+\ldots+d_{k_{p(k)}{ }^{n}}^{\circ}$

Where at least one equal sign holds for all possible paths from vertex $i$ to vertex $n$.Let $f^{\circ}(i)$ be the length of the shortest path from vertex $i$ to vertex $n$ in network $G(V, E)$ with $\left\{d_{i j}^{\circ} \mid\langle i, j\rangle \in E\right\}$. Therefore from (7), we get $f^{\circ}(i)=d_{i i_{1}}^{\circ}{ }^{\circ} d_{i_{1} i_{2}}^{\circ}+\ldots+d_{i_{m(i)}{ }^{n}}^{\circ}$. similarly,we obtain $f^{\circ}(j)=d_{j j_{1}}^{\circ} d_{j_{1} j_{2}}^{\circ}{ }^{+}{ }^{+} d_{j_{m(j)}{ }^{n}}$ We rewrite (1) as follows: for any fixed $i, f(i) \leq d_{i j}+f(j), \forall i<j,\langle i, j\rangle \in E$,

Where at least one equal sign holds. Then

$$
d_{i i_{1}}+d_{i_{i i_{2}}}+\ldots+d_{i_{n i(i)}{ }^{n}} \leq d_{i j}+d_{j j_{1}}+d_{j_{j} j_{2}}+. .+d_{j_{n(j)} n}, \forall i<j,\langle i, j\rangle \in E
$$

Where at least one equal sign holds. The DM should choose appropriate values for parameters to satisfy

$$
\delta_{i_{1}}+\delta_{i i_{2}}+. . \delta_{l_{i, i}{ }^{n}} \leq \delta_{j}+\delta_{j_{1}}+\delta_{j_{1} j_{2}}+. .+\delta_{j_{m j}{ }^{n}}, \forall i<j,\langle i, j\rangle \in E
$$

where at least one equal sign holds. from (9) and (10), we obtain

$$
\begin{aligned}
& \widetilde{d}_{i i_{1}} \oplus \widetilde{d}_{i i_{2}} \oplus . . \oplus \widetilde{d}_{i_{n(i)} n} \leq \widetilde{d_{i j}} \widetilde{d}_{j_{1}} \oplus . \oplus \widetilde{d}_{j_{n(j)} n} \\
& \forall i<j,\langle i, j\rangle \in E,
\end{aligned}
$$

Where at least one $\approx$ holds. From definition we can write

$$
\begin{aligned}
& d_{i_{i_{1}}}^{\circ} d_{i_{1} i_{2}}^{\circ}+\ldots+d_{i_{m(i)}{ }^{n}}^{\circ} \leq d_{j j_{1}}^{\circ} d_{j_{1} j_{2}}^{\circ}{ }^{\circ}+ \\
& d_{j_{m(j)}{ }^{n}}^{\circ}, \forall i<j,\langle i, j\rangle \in E
\end{aligned}
$$

Where at least one equal sign holds.

From (8) and (12), the DP recusion of the first type of shortest -path problem in the fuzzy sense can be given by $f^{\circ}(i)=\min _{i<j}\left\{d_{i j}^{\circ}+f^{\circ}(j) \mid\langle i, j\rangle \in E\right\}$, and $f^{\circ}(n)=0$.

Now consider the fuzzy case. We look for inequalities that satisfy (10).

When $\mathrm{i}=1$,

$$
\begin{gathered}
d_{13}+f(3)<d_{12}+f(2), \\
\text { i.e, } d_{13}+d_{36}+d_{68}+d_{89}<d_{12}+d_{27}+d_{79}, \\
d_{13}+f(3)<d_{14}+f(4), \text { i.e. } \\
d_{13}+d_{36}+d_{68}+d_{89}
\end{gathered}
$$$$
d_{14}+d_{46}+d_{68}+d_{89}
$$ 
or $d_{14}+f(4)<d_{12}+f(2)$, i.e.

$d_{14}+d_{46}+d_{68}+d_{89}<d_{12}+d_{27}+d_{79}$

When $\mathrm{i}=2$,

$$
\begin{aligned}
& d_{27}+f(7)<d_{25}+f(5), \text {,i.e. }< \\
& d_{27}+d_{79}<d_{25}+d_{58}+d_{89},
\end{aligned}
$$

When $\mathrm{i}=3$,

$$
\begin{array}{r}
d_{36}+f(6)<d_{35}+f(5), \text { i.e. } \\
d_{36}{ }^{+} d_{68}+d_{89}<d_{35}+d_{58}+d_{89},
\end{array}
$$

When $\mathrm{i}=5$,

$$
\begin{gathered}
d_{58}+f(8)<d_{57}+f(7) \text {,i.e. } \\
d_{58}+d_{89}<d_{57}+d_{79} .
\end{gathered}
$$

Then, the parameters of (10) based on the above inequalities are derived as

$$
\begin{aligned}
& \delta_{13}+\delta_{36}+\delta_{68}+\delta_{89}<\delta_{12}+\delta_{27}+\delta_{79} \\
& \delta_{13}+\delta_{36}+\delta_{68}+\delta_{89} \\
& \delta_{14}+\delta_{46}+\delta_{68}+\delta_{89} \\
& \delta_{14}+\delta_{46}+\delta_{68}+\delta_{89}<\delta_{12}+\delta_{27}+\delta_{79} \\
& \delta_{27}+\delta_{79}<\delta_{25}+\delta_{58}+\delta_{89}, \\
& \delta_{36}+\delta_{68}+\delta_{89}<\delta_{35}+\delta_{58}+\delta_{89}, \text { and } \\
& \delta_{58}+\delta_{89}<\delta_{57}+\delta_{79} .
\end{aligned}
$$

If the DM choose the values of parameters: $\delta_{12}=14$, $\delta_{13}=12, \quad \delta_{14}=10, \quad \delta_{25}=9, \quad \delta_{27}=11, \quad \delta_{35}=7$, $\delta_{36}=5, \quad \delta_{46}=6, \quad \delta_{57}=8, \quad \delta_{58}=7, \quad \delta_{68}=8$, $\boldsymbol{\delta}_{79}=11, \boldsymbol{\delta}_{89}=10$,to satisfy the condition in (13) then the fuzzy numbers can be determined as follows : $\widetilde{d}_{12}=(355-5,355,355+19), \widetilde{d}_{13}=(305-6,305$, $305+18), \widetilde{d}_{14}=(243-3,243,243+13)$, $\widetilde{d}_{25}=(116-3,116,116+12), \quad \widetilde{d}_{27}=(245-4,245$,

245+15), $\widetilde{d}_{35}=(140-3,140,140+10)$,

$\widetilde{d}_{36}=(78-3,78,78+8), \widetilde{d}_{46}=(144-4,144,144+10)$,

$\widetilde{d}_{57}=(181-3,181,181+11)$,

$\widetilde{d}_{58}=(160-3,160,160+10), \quad \widetilde{d}_{68}=(168-4,168$,

168+12), $\widetilde{d}_{79}=(116-5,116,116+16)$,

$\widetilde{d}_{89}=(132-2,132,132+12)$.

Therefore we can find the defuzzified values as

$d_{12}^{\circ}=358.5, \quad d_{13}^{\circ}=308, \quad d_{14}^{\circ}=245.5$,

$d_{25}^{\circ}=118.25, \quad d_{27}^{\circ}=247.75, \quad d_{35}^{\circ}=141.75$,

$d_{36}^{\circ}=79.25, \quad d_{46}^{\circ}=145.5, \quad d_{57}^{\circ}=183$,

$d_{58}^{\circ}=161.75, \quad d_{68}^{\circ}=170, \quad d_{79}^{\circ}=118.75$,

$d_{89}^{\circ}=134.5$.The fuzzy network $G=(V, E)$

with $\left\{d_{i j}^{\circ} \mid\langle i, j\rangle \in E\right\}$.

Where

$$
\begin{aligned}
& f^{\circ}(1)=d_{13}^{\circ}+f^{\circ}(3)=d_{13}^{\circ}+d_{36}^{\circ}+f^{\circ}(6)= \\
& d_{13}^{\circ}+d_{36}^{\circ}+d_{68}^{\circ}+f^{\circ}(8)=d_{13}^{\circ}+d_{36}^{\circ}+d_{68}^{\circ}+ \\
& d_{89}^{\circ},
\end{aligned}
$$

The fuzzy shortest path is is 1-3-6-8-9 with length 691.75.The shortest path in the fuzzy sense is longer than the crisp shortest path by

$$
\frac{f^{\circ}(1)-f(1)}{f(1)} \times 100=1.2811 \% \text {. }
$$

\section{CONCLUSIONS}

In the present paper the arc lengths are considered as uncertain and which are characterized by triangular fuzzy number. For computation of shortest path Bellman Dynamic Programming technique is adopted. As a real life example Rajasthan State Roadways Transport Network has been considered and with the help of dynamic programming recursion formulation shortest path in this network is computed both in 
crisp and fuzzy environments. The percentage difference is very less and which is acceptable.

\section{REFERENCES}

1. J. S. Yao and K. M. Wu, "Ranking fuzzy numbers based on decomposition principle and signed distance", Fuzzy Sets and Systems, Vol.116, , p.275-288, 2000.

2. X.wang and E.E.Kerre, "Reasonable properties for the ordering of fuzzy quantities (I)," Fuzzu Sets and Systems, Vol. 118, p. 375-385, 2001.

3. X.wang and E.E.Kerre, "Reasonable properties for the ordering of fuzzy quantities (II)," Fuzzu Sets and Systems, Vol. 118, p. 387-405, 2001.

4. D. Dubois and H. Prade, Fuzzy Sets and Systems, Academic Press, New York, 1980.

5. S. Okada and T. Soper, "A shortest path problem on a network with fuzzy are lengths", Fuzzy Sets and Systems, Vol. 109, p. 129-140, 2000.

6. C. M. Klein, "Fuzzy Shortest Paths", Fuzzy Sets and Systems, Vol. 39, 1991E, pp.27-41, '1991.

7. A. Kauffman and M. M. Gupta, Introduction to Fuzzy Arithmetic Theory and Applications, van Nostrand Reinhold, New York, 1991.
8. E. Lawler, Combinatorial Optimization: Networks and Mastoids, Holt, Reinehart and Winston, New York, 1976.

9. Jing-Shing Yao and Feng-Tse Lin, "Fuzzy Shortest-Path Network Problems with Uncertain Edge Weights," Journal of Information Science and Engineering, Vol. 19, p.329-351 , 2003.

10. K. Lin and M. Chen, "The fuzzy shortest path problem and its most vital arcs", Fuzzy Sets and Systems, Vol. 58, pp.343-353, 1994.

11. M. Mares and J. Horak, "Fuzzy quantities in networks", Fuzzy Sets and Systems, Vol.10, 1983, p.135-155.

12. S.Chanas and W. Kolodziejczyk, "Masimum flow in a network with fuzzy arc capacities," Fuzzy Sets and Systems, Vol.8, p.165-173, 1982.

13. T, Cormen, C. Leiserson, and R. Rivest, 'Introduction to Algorithms,' McGraw Hill Book Company, Mass., 1993.

14. S.Okada and M.Gen, 'Fuzzy shortest path problem ,' Comput. Industrial Eng.,27, p.465$468,1994$. 